\title{
EVALUACIÓN DE La ASTENIA EN ONCOLOGÍA. APLICACIÓN DEL CUESTIONARIO PERFORM
}

\author{
ASSESSMENT OF FATIGUE IN ONCOLOGY. IMPLEMENTATION OF THE PERFORM \\ QUESTIONNAIRE
}

\author{
César A. Rodríguez, 1,3, Maribel Ruiz², Rosa Alonso3, Fernanda Viotti ${ }^{4}$, Mariam El-Hayaj \\ Elvira del Barco ${ }^{1,3,4}$ y Juan J. Cruz $z^{1,3,4}$ \\ ${ }^{1}$ Servicio de Oncología Médica. Hospital Universitario de Salamanca. \\ ${ }^{2}$ Sección de Oncología Médica. Hospital Río Carrión de Palencia. \\ ${ }^{3}$ Master Universitario en Cuidados Paliativos y Tratamiento de Soporte en el Enfermo Oncológico. Universidad \\ de Salamanca. \\ ${ }^{4}$ Título Propio de Experto Universitario en Cuidados Paliativos y Tratamiento de Soporte en el Enfermo Onco- \\ lógico. Universidad de Salamanca.
}

Resumen

La astenia relacionada con el cáncer constituye uno de los problemas más frecuentes y con mayor repercusión sobre la calidad de vida en los pacientes oncológicos, y a menudo persiste durante meses o años después de finalizado el tratamiento. Su etiología y fisiopatología son complejas y en la mayoría de los casos no bien conocidas, existiendo múltiples interacciones con otras entidades clínicas relacionadas con el cáncer. En cuanto al abordaje terapéutico, debe ser individualizado, incluyendo el tratamiento de los factores contribuyentes, la educación del paciente, el establecimiento de medidas generales, la administración de fármacos y las intervenciones no farmacológicas, entre las que destaca el ejercicio físico. Con el fin de facilitar la detección y evaluación periódica de la astenia en el paciente oncológico dentro de la práctica clínica habitual, se han desarrollado una serie de herramientas que clásicamente pueden agruparse en dos tipos: unidimensionales y multidimensionales. Sin embargo, una de las cuestiones más importantes de las escalas de evaluación de calidad de vida en general y de la astenia en particular es que su desarrollo y validación se haya llevado

\section{Abstract}

Fatigue-related cancer is one of the most frequent problems and one of the symptoms with more impact in the quality of life in patients with cancer; and often it persists during months or years after the treatment has finished. The etiology and physiopathology are complex and, in most of cases, not well known, existing multiple interactions with other clinical entities related with cancer. The therapeutical approach must be individualized, including the treatment of contributing factors, education of the patient, the implementation of general therapeutic reccomendations, pharmacological treatment and non-pharmacologic interventions, specially exercise. With the aim of detect and assess fatigue-related cancer in the clinical practice, some scales and questionnaires have been developed. Classicaly the have been classified as unidimensional and multidimensional questionnaires. However, one of the most important questions regarding the quality of life scales, and in particular fatigue scales, is that it's design, development and validation must be performed in the social and cultural context of the population in which the questionnaire is going to be implemented.

\section{Correspondencia:}

César A. Rodríguez Sánchez

Servicio de Oncología Médica. Hospital Universitario de Salamanca

Paseo de San Vicente 58 - 37007 Salamanca (Spain)

E-mail: crodriguez@oncologiasalamanca.org 
a cabo en el contexto sociocultural en que se aplicarán. El cuestionario Perform es el único desarrollado y validado íntegramente en población oncológica española.

Palabras clave: Astenia, cáncer, superviventes, cuestionario Perform.
The Perform Questionnaire is the only scale for the assessment of fatigue-related cancer developed and validated completely in a Spanish population.

Keywords: Fatigue-Related Cancer, Fatigue, survivor, Perform Questionnaire.

\section{INTRODUCCIÓN}

La astenia relacionada con el cáncer se define como "una sensación angustiosa, persistente y subjetiva de cansancio o agotamiento relacionada con el cáncer o su tratamiento, que no es proporcional con la actividad realizada y que interfiere con las funciones habituales"(1). A diferencia de la astenia experimentada por un individuo sano, es más intensa, más angustiosa y no se alivia con el descanso.

Se trata de uno de los síntomas con mayor prevalencia en el paciente oncológico, de manera que el $95 \%$ de los pacientes que reciben quimioterapia $y / o$ radioterapia presentan astenia en mayor o menor grado $^{(2)}$. Además, hasta en un 17\%-30\% de los supervivientes persiste durante meses o incluso años después de finalizar el tratamiento ${ }^{(3,4)}$.

Por otra parte, la astenia se asocia a un detrimento en las actividades físicas, psicológicas y sociales, lo que impacta desfavorablemente en la calidad de vida. Su influencia sobre este aspecto es mayor que la de otros síntomas, como las náuseas, el dolor o la depresión ${ }^{(2)}$.

En un estudio acerca de la percepción de la astenia en pacientes con cáncer en España, un 97,8\% de los pacientes refería haber experimentado astenia a lo largo del tratamiento oncológico, lo que afectaba notablemente su estado de ánimo. Además, la astenia era considerada el síntoma más limitante para la mayoría de los pacientes, independientemente del sexo o del tipo de tumor ${ }^{(5)}$.

\section{FISIOPATOLOGÍA}

Los mecanismos fisiopatológicos implicados en la aparición de la astenia relacionada con el cáncer no están completamente aclarados. Probablemente estén relacionados con una alteración en la regulación de varios sistemas y a la asociación de factores contribuyentes (ver figura 1), cuyo efecto puede ser variable en función del individuo, del las diferentes fases de la enfermedad o del tipo de tumor. Entre los mecanismos descritos se encuentran alteraciones en la regulación de la serotonina, disfunción del eje hipotálamo-hipófisis-adrenal, alteraciones del ritmo circadiano, alteraciones en el metabolismo muscular/ATP, activación de vías vagales aferentes y alteración en la regulación de citoquinas ${ }^{(6)}$.

\section{a.- Alteración en la regulación de la serotonina}

El incremento en los niveles de serotonina (5-hidroxitriptamina o 5-HT) a nivel cerebral y/o el aumento en la estimulación de los receptores 5-HT como consecuencia del cáncer o de su tratamiento constituye uno de los posibles mecanismos responsables de la astenia. Esta alteración podría ser debida a la actividad de las citoquinas proinflamatorias, como interleuquina (IL)- $1 \beta$, interferon (IFN)- $\alpha$, IFN- $\gamma$ y TNF- $\alpha$, que estimulan la indolamina-2,3-dioxigenasa y alteran así el metabolismo de $5-\mathrm{HT}^{(6)}$. 
Figura 1. Fisiopatología de la astenia relacionada con el cáncer

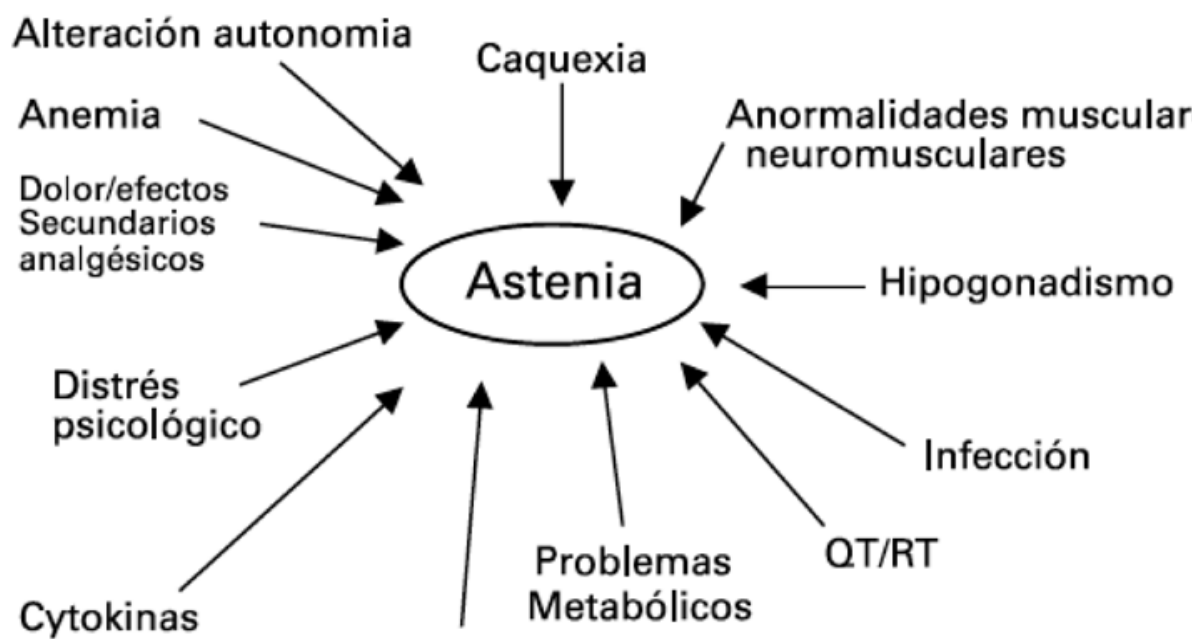

Deshidratación

\section{b.- Disfunción del eje hipotálamo- hipófisis-adrenal}

El eje hipotálamo-hipófisis-adrenal (HHA) regula la liberación de cortisol en respuesta al estrés físico o psicosocial. La astenia se asocia a niveles bajos de cortisol, que podrían ser debidos a una supresión directa del eje HHA por el tratamiento antitumoral (por ejemplo, corticoides, radioterapia o algunos tipos de quimioterapia) o a cambios en 5-HT que den lugar a una menor estimulación de los receptores $5-\mathrm{HT}_{1 \mathrm{~A}}$, responsables de señalizar la liberación de la hormona liberadora de corticotropina $(\mathrm{CRH})^{(7)}$.

\section{c.- Alteración del ritmo circadiano}

En los pacientes con cáncer se han demostrado diferentes alteraciones en el ritmo circadiano, incluyendo cambios en los ritmos hormonales (por ejemplo, la secreción de cortisol y melatonina), los procesos metabólicos (por ejemplo, la temperatura o los niveles circulantes de proteínas), el sistema inmune (por ejemplo, los niveles de leucocitos y neutrófilos circulantes) y los patrones de actividaddescanso $^{(8)}$. El ritmo circadiano se ve afectado fundamentalmente en pacientes con enfermedad avanzada, mientras que en estadios tempranos esta afectación es escasa o nula.

\section{d.- Alteraciones en la regulación del metabolismo muscular/ATP}

El ATP supone la mayor fuente de energía para la contracción muscular. En los pacientes con cáncer la anorexia-caquexia puede dar lugar a un déficit en la capacidad de regeneración de ATP en el músculo esquelético, debido a una alteración del metabolismo proteico muscular ${ }^{(6)}$.

\section{e.- Activación de vías vagales aferentes}

El cáncer o sus tratamientos pueden inducir la liberación periférica de agentes neuroactivos que estimulan fibras vagales aferentes, provocando la supresión de la actividad de la musculatura somática y la inducción de una "conducta de enferme-

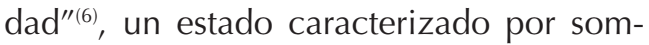
nolencia, pérdida de apetito, dificultad 
para la concentración y malestar general. Aunque los resultados de algunos estudios con animales apoyan este mecanismo, existe escasa evidencia en humanos.

\section{f.- Alteración en la regulación de citoquinas}

Las citoquinas proinflamatorias, como TNF- $\alpha$, IL-1 $\beta$ o IFN- $\alpha$, participan en la mayoría de los mecanismos propuestos como desencadenantes de la astenia asociada al cáncer, especialmente en supervivientes ${ }^{(9)}$. El cáncer y sus tratamientos se asocian con una elevación de los niveles plasmáticos de citoquinas, implicadas en el desarrollo de anemia, caquexia, anorexia, fiebre, infecciones y depresión, lo que favorece la aparición de astenia.

\section{OTROS FACTORES CONTRIBUYENTES}

Es importante señalar que la astenia en el paciente oncológico a menudo está acompañada por una serie de factores que contribuyen a su desarrollo, como por ejemplo la anemia, la caquexia, la depresión o los trastornos del sueño.

\section{a.- Anemia}

Varios estudios han evidenciado una relación entre anemia y astenia(10,11). El mecanismo por el cual la anemia puede provocar astenia en el paciente con cáncer no está totalmente aclarado, aunque podría estar relacionado con la alteración funcional de diferentes órganos como consecuencia de la hipoxia(6).

\section{b.- Caquexia}

La caquexia tumoral implica una pérdida de tejido adiposo y masa muscular esquelética, que da lugar a anorexia, pérdida de peso, astenia y disminución de la supervivencia. La etiología de la ca- quexia tumoral es compleja e implica no solamente una reducción en la ingesta de nutrientes, sino también alteraciones metabólicas provocadas por el tumor, incluyendo un aumento del catabolismo proteico y una reducción de la síntesis de proteínas en el músculo esquelético. La proteolisis es inducida por citoquinas y otras sustancias catabólicas, como el factor movilizador de lípidos y el factor inductor de proteolisis.

\section{c.- Depresión}

Aunque algunos autores han propuesto una etiología común en el desarrollo de la depresión y la astenia, actualmente no existe suficiente evidencia científica para afirmar que una condición predice la otra ${ }^{(12)}$.

\section{d.- Trastornos del sueño}

Los trastornos del sueño pueden ser causados por diferentes mecanismos, como la disfunción del eje HHA, las alteraciones en el ritmo circadiano o en el metabolismo de la serotonina y los cambios en la expresión de citoquinas ${ }^{(6)}$, todos ellos implicados a su vez en la aparición de astenia.

\section{EI TRATAMIENTO DE LA ASTENIA EN EL PACIENTE ONCOLÓGICO}

Tal y como se ha señalado anteriormente, la astenia relacionada con el cáncer es un síndrome multidimensional, en el que participan diferentes mecanismos fisiopatológicos. En los pacientes con cáncer avanzado o recurrente, un tratamiento antineoplásico apropiado con frecuencia logra una mejoría de la astenia y otros síntomas. Sin embargo, en aquellos pacientes con astenia persistente durante el tratamiento o tras la finalización del mismo es necesario emplear otras estrategias, inte- 


\section{Figura 2. Algoritmo de actuación en la astenia en supervivientes}

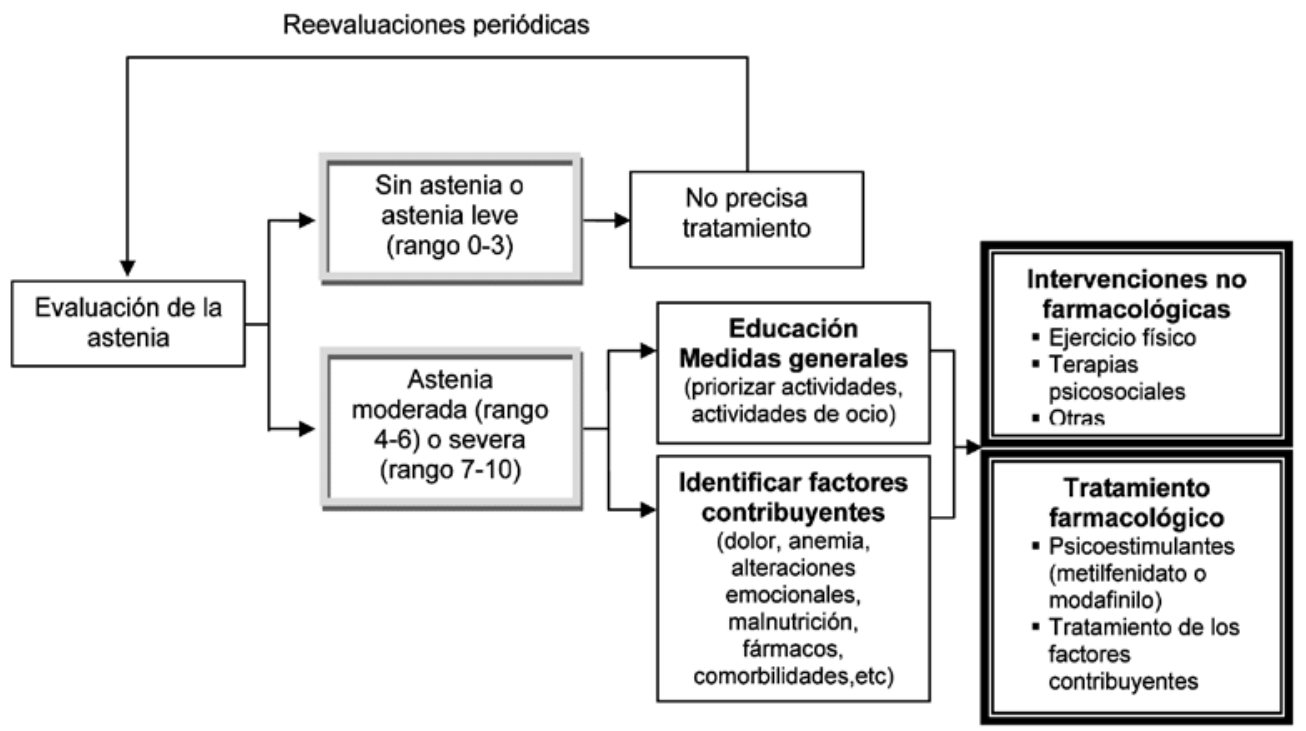

gradas dentro de una actuación multidisciplinar que incluya tanto la administración de fármacos como otras intervenciones no farmacológicas (ver figura 2).

El primer paso en el tratamiento de la astenia en el paciente oncológico es la identificación de posibles factores contribuyentes, potencialmente reversibles, como dolor, alteraciones emocionales (ansiedad o depresión), anemia, malnutrición, fármacos administrados, abuso de alcohol u otras sustancias o comorbilidades no relacionadas con el cáncer, como los trastornos endocrinológicos (por ejemplo, hipotiroidismo, hipogonadismo o insuficiencia suprarrenal), infecciones, cardiopatías, patología pulmonar, renal, neurológica o gastrointestinal.

Por otra parte, es recomendable informar al paciente acerca de las causas y evolución esperable de los síntomas. Aunque en un subgrupo de los superviventes puede persistir un grado de astenia que interfiera con las actividades diarias, la mayoría de los pacientes experimentan una progresiva mejoría y recuperan sus niveles normales de energía.
También puede ser útil adoptar una serie de medidas generales, como la planificación previa de las actividades, estableciendo prioridades en función de los recursos energéticos ${ }^{(13)}$, o la realización de actividades de ocio que favorezcan la distracción ${ }^{(14)}$ (por ejemplo los juegos, la música o la lectura).

\section{a.- Tratamiento farmacológico}

- Agentes eritropoyéticos

Varios estudios han demostrado que su administración como tratamiento de la anemia en pacientes oncológicos reduce la necesidad de transfusiones y mejora la calidad de vida, disminuyendo los niveles de $\operatorname{astenia}^{(11,15,16)}$. No obstante, ante la publicación en los últimos años de datos controvertidos sobre su seguridad, se recomienda considerar el índice riesgo/beneficio individual antes de plantear su utilización y restringir su uso a pacientes en tratamiento con quimioterapia con intención no curativa. 
- Psicoestimulantes

Los psicoestimulantes proporcionan sensación de bienestar, disminuyen la astenia y potencian la atención. Poseen la ventaja de su rápido efecto, aunque también pueden provocar efectos secundarios, como insomnio, euforia o labilidad emocional.

El metilfenidato es el agente empleado con más frecuencia, aunque su uso sigue siendo controvertido por la heterogeneidad de los resultados obtenidos en los diferentes estudios $^{(17-21)}$ y posibilidad de efectos secundarios, como ansiedad o disminución del apetito. Sin embargo, algunos ensayos han mostrado resultados favorables cuando se administra en pacientes con astenia persistente tras la finalización del tratamiento $^{(21,22)}$.

El modafinilo ha demostrado ser superior a placebo en el tratamiento de pacientes en tratamiento con quimioterapia y que presenten astenia severa, mientras que no se ha observado beneficio en aquellos pacientes con astenia leve o modera$\mathrm{da}^{(23)}$. También ha mostrado resultados prometedores en superviventes con astenia persistente, por lo que podría valorarse su administración en este escenario.

- Antidepresivos

Dos estudios han evaluado el papel de la paroxetina, un inhibidor selectivo de la captación de serotonina, en el tratamiento de la astenia relacionada con el cáncer sin objetivarse beneficio respecto a placebo tras 8 semanas de tratamiento ${ }^{(24,25)}$, a pesar de obtenerse mejoría de la sintomatología depresiva. Estos resultados sugieren la participación de distintos mecanismos en el desarrollo de la astenia y la depresión.
También se han realizado ensayos clínicos con bupropion, un inhibidor selectivo mixto de la recaptación de noradrenalina y dopamina, evidenciándose mejoría de la astenia tras 2-4 semanas de terapia ${ }^{(26,27)}$. Sin embargo, la evidencia sobre su eficacia en supervivientes es limitada.

- Corticoesteroides

La administración de corticoesteroides puede mejorar la calidad de vida y reducir la astenia en pacientes con cáncer en estadios avanza$\operatorname{dos}^{(28)}$, actuando probablemente a través de la inhibición de citoquinas proinflamatorias. La dosis y el tipo de corticoesteroide ideal en estos casos no está bien establecida, aunque en la mayoría de los ensayos se utilizan 20-40 mg al día de prednisona o su equivalente. Provocan un efecto rápido que suele durar entre 2 y 4 semanas, no recomendándose su empleo durante periodos más prolongados dado el riesgo de efectos adversos.

\section{- Otros}

El uso de progestágenos, como el acetato de megestrol, se ha asociado con una disminución de la astenia y un aumento del apetito $y$ de la sensación de bienestar en pacientes con cáncer avanzado ${ }^{(29)}$. Sin embargo, cuando se realiza un análisis combinado de los resultados de diferentes estudios no se evidencia beneficio respecto a placebo ${ }^{(21)}$, por lo que actualmente no se aconseja su uso para el tratamiento de la astenia en la práctica clínica habitual. Se han publicado recientemente datos prometedores sobre el extracto de guarana (Paullina cupana) en el tratamiento de la astenia de pacientes con cáncer de mama en trata- 
miento con quimioterapia, perfilándose como una alternativa eficaz, poco tóxica y de bajo coste ${ }^{(30)}$.

Otros fármacos, como la L-carnitina o el donezepilo también han sido evaluados, sin mostrar un claro beneficio en el control de la astenia.

\section{b.- Tratamiento no farmacológico}

- Ejercicio físico

Actualmente representa la alternativa no farmacológica más avala$\mathrm{da}^{(31,32)}$ por la evidencia científica. El ejercicio físico moderado, practicado regularmente, puede reducir la ansiedad y la depresión, mejorar la imagen corporal y aumentar la tolerancia a la actividad física. Aunque no existe una conclusión firme sobre el tipo de ejercicio más ${ }^{(33)}$ efectivo (aeróbico y/o resistencia), su frecuencia o intensidad, generalmente se recomienda realizar un ejercicio aeróbico moderado, durante 20-60 minutos de 3 a 5 veces por semana, y ejercicios de resistencia, con 3 series de 8 a 12 repeticiones por ejercicio, practicados 2 o 3 veces por sema$\mathrm{na}^{(34)}$. No obstante, debe limitarse la actividad física en pacientes frágiles o con patología cardiorrespiratoria, y aconsejarse precaución en aquellos con metástasis óseas, trombopenia, anemia o infecciones activas.

\section{- Terapias psicosociales}

Se han investigado una amplia variedad de intervenciones psicosociales, que pueden agruparse en terapias de apoyo grupal, psico-educacional o cognitivo-conductual ${ }^{(35-37)}$. A pesar de la herogeneidad de las medidas evaluadas, los resultados de diferentes estudios sugieren que este tipo de intervenciones benefician al paciente con cáncer mejorando la $\operatorname{astenia}^{(35,36)}$. Sin embargo, los resultados de un reciente estudio ${ }^{(37)}$ muestran que la terapia cognitivoconductual no aporta un beneficio adicional sobre el ejercicio físico en el tratamiento de la astenia del paciente oncológico.

Otras estrategias no farmacológicas incluyen yoga, pilates, meditación, terapia del sueño o soporte nutricional ${ }^{(38)}$.

\section{LA EVALUACIÓN DE LA ASTENIA EN PACIENTES CON CÁNCER}

En el paciente oncológico debe valorarse la presencia de astenia desde la primera visita, realizando posteriormente reevaluaciones periódicas con el fin de iniciar, si es necesario, un tratamiento precoz y efi$\mathrm{caz}^{(1)}$. Sin embargo, el hecho de que se trate de una experiencia subjetiva dificulta notablemente su valoración. En la práctica clínica habitual, una forma sencilla de detectar la presencia e intensidad de la astenia puede ser el empleo de una escala numérica, el 0 al 10, en la que una puntuación de 0 indica la ausencia de astenia, el rango de 1 a 3 señala la presencia de astenia leve que no precisa tratamiento, y los rangos de 4 a 6 y de 7 a 10 indican astenia moderada o severa, respectivamente, que requiere una evaluación más amplia y una intervención terapéutica.

Gran parte de las herramientas que evalúan la astenia en los pacientes con cáncer se centran únicamente en la identificación de su presencia e intensidad, mientras que otras examinan también su impacto sobre la actividad física, la función cognitiva y la situación socio-emocional. Teniendo en cuenta este aspecto podemos diferenciar dos tipos de herramientas ${ }^{(39,40)}$ :

\section{1.- Unidimensionales}

Son las utilizadas con más frecuencia en la práctica clínica diaria, debido a su 
sencillez y brevedad. Sin embargo, se limitan a valorar la presencia/ausencia de astenia y su intensidad, por lo que pueden omitir ciertos aspectos importantes relacionados con la astenia, más allá de su impacto en la actividad física.

A menudo se trata de evaluaciones extraídas de cuestionarios más amplios, como Sympton Distress Scale, Rotterdam Symptoms Checklist, European Organization for Research and Treatment of Cancer (EORTC) QLC-C30, Medical Outcomes Study 36-Item Short-Form Health Survey (SF36), MD Anderson Symptoms Inventory y Zung Self-Rating Depression Scale. Se centran principalmente en detectar la presencia o ausencia de astenia, y no suelen referirse a su intensidad o impacto sobre los diferentes aspectos de la vida diaria.

Existen además escalas con un solo ítem desarrolladas específicamente para evaluar la presencia e intensidad de la astenia relacionada con el cáncer, como es el caso de la Visual Analog Fatigue Scale $(\mathrm{VAFS})^{(41)}$. Esta escala es útil también para individuos sanos, lo que ofrece la posibilidad de realizar comparaciones con la astenia del paciente oncológico; además, su facilidad de uso permite monitorizar la astenia en diferentes momentos del día.

Entre los cuestionarios con múltiples items que han sido validados en pacientes con cáncer, destacan Brief Fatigue Inventory (BFI), que mide la intensidad de la astenia en las 24 horas previas, o Cancer-Related Fatigue Distress Scale, que evalúa el impacto de la astenia en la última semana.

\section{2.- Multidimensionales}

Tienen en cuenta además otros aspectos relacionados con la astenia, como su impacto sobre el comportamiento del individuo o su función cognitiva, física y afectiva. Esta amplia valoración generalmente supone una mayor complejidad del cuestionario, lo que reduce su empleo en la práctica clínica diaria. Entre ellas se encuentran diferentes tipos de escalas, como Multidimensional Fatigue Inventory (MFI), Functional Assessment of Cancer TherapyFatigue scale (FACT-F), Multidimensional Fatigue Symptom Inventory (MFSI), Revised Piper Fatigue Scale (PFS) o Fatigue Sympton Inventory (FSI).

Una de las limitaciones que presentan estas herramientas es su desarrollo en contextos culturales ajenos al entorno en el que se pretenden aplicar. Por ello es necesario crear instrumentos que permitan identificar y cuantificar la astenia relacionada con el cáncer en el área geográfica en la que van a ser utilizados, asegurando además su validez y fiabilidad psicométrica. Con este fin se llevó a cabo el Proyecto PERFORM ${ }^{(42)}$, que ha elaborado el primer cuestionario desarrollado y validado en población española (Tabla 1), aplicable en la práctica clínica diaria y que, en el momento actual constituye una herramienta sólida elaborada con una base metodológica reconocida y reproductible.

\section{EL CUESTIONARIO PERFORM EN EVALUACIÓN DE LA ASTENIA RELACIONADA CON EL CÁNCER}

Como se ha comentado anteriormente, no se conocía, hasta la elaboración del cuestionario Perform un instrumento para evaluar la astenia relacionada con el cáncer que hubiera sido desarrollado y validado íntegramente en España, lo que significaba que ciertos aspectos, relevantes para nuestros pacientes oncológicos, no estarían siendo valorados al utilizar cuestionarios procedente de otros contextos socioculturales. Estos cuestionarios reflejan y evalúan un determinado concepto de CVRS que puede no encajar con la realidad de los pacientes españoles, porque el concepto de CVRS puede diferir según el entorno cultural ${ }^{(43)}$. 


\section{Tabla 1. Cuestionario PERFORM para la evaluación de la astenia relacionada con el cáncer}

\begin{tabular}{|c|c|c|c|c|c|c|}
\hline $\begin{array}{l}\text { EN LAS ÚLTIMAS } 2 \text { SEMANAS } \\
\text { ¿Con qué frecuencia se ha encontrado con esta situación o síntoma? }\end{array}$ & 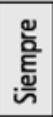 & 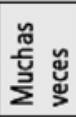 & $\frac{\pi}{\mathbb{m}}$ & & ֻ & \\
\hline $\begin{array}{l}\text { 1. Realizar un ejercicio mínimo me ha supuesto un cansancio } \\
\text { desproporcionado. }\end{array}$ & 1 & 2 & 3 & 4 & 5 & \\
\hline $\begin{array}{l}\text { 2. El cansancio que he notado (debido a la enfermedad o su tratamiento) } \\
\text { era muy diferente al cansancio "normal". }\end{array}$ & 1 & 2 & 3 & 4 & 5 & \\
\hline 3. He estado cansado/a todo el día. & 1 & 2 & 3 & 4 & 5 & \\
\hline 4. Me he pasado todo el día sentado/a a causa de mi cansancio. & 1 & 2 & 3 & 4 & 5 & \\
\hline $\begin{array}{l}\text { 5. Cuando he estado cansado/a he tenido que interrumpir lo que estaba } \\
\text { haciendo y descansar para poder sequir. }\end{array}$ & 1 & 2 & 3 & 4 & 5 & \\
\hline 6. Me he notado muy lento haciendo mi actividad diaria. & 1 & 2 & 3 & 4 & 5 & \\
\hline $\begin{array}{l}\text { 7. He necesitado ayuda para hacer las tareas de casa, a causa de mi } \\
\text { cansancio. }\end{array}$ & 1 & 2 & 3 & 4 & 5 & \\
\hline 8. Me he encontrado cansado/a en el trabajo, y eso me ha hecho sentir fatal. & 1 & 2 & 3 & 4 & 5 & No procede \\
\hline 9. En general creo que mi vida ha empeorado a causa del cansancio. & 1 & 2 & 3 & 4 & 5 & \\
\hline 10. He sentido que me estoy viniendo abajo a causa de mi cansancio. & 1 & 2 & 3 & 4 & 5 & \\
\hline 11. Siento que el cansancio me ha impedido hacer una vida normal. & 1 & 2 & 3 & 4 & 5 & \\
\hline 12. He dejado de hacer lo que me gusta a causa de mi cansancio. & 1 & 2 & 3 & 4 & 5 & \\
\hline
\end{tabular}

\section{(a cumplimentar por el personal sanitario)}

\section{Para obtener la puntuación global del cuestionario sume la puntuación de todas las preguntas del cuestionario.}

Suma total $=$

Bajo estas circunstancias nació el Proyecto PERFORM, cuyo objetivo era desarrollar el primer cuestionario español para evaluar las percepciones sobre la astenia relacionada con el cáncer en pacientes oncológicos, para uso en la práctica clínica diaria.

El proceso de construcción del Cuestionario PERFORM empezó en el año 2004 y fue desarrollado en diversas fases sucesivas, que permitieron que el cuestionario cumpliera con los estándares y requisitos establecidos por Comité Científico Asesor del Medical Outcomes Trust (MOT) ${ }^{(44)}$. Las fases del desarrollo del cuestionario se describen a continuación:

\section{Primera fase (2004)}

En una primera fase se obtuvo el contenido del cuestionario y la definición de las características básicas del mismo mediante la revisión de los principales cuestionarios existentes para la evaluación de la astenia relacionada con el cáncer. Adicionalmente, se realizó una reunión con expertos clínicos y expertos en la medida de resultados en salud para determinar las características que debería tener el cuestionario. También se realizaron grupos de discusión con pacientes oncológicos en distintos lugares de España. Del análisis de la información obtenida a partir de las anteriores iniciativas, se generó un listado inicial de ítems potenciales para el cuestionario que evaluaban la percepción de los pacientes oncológico respecto a la astenia ${ }^{(45)}$.

\section{Segunda fase (2004 - 2005)}

Durante la fase de construcción de medidas centradas en el paciente es ha- 
bitual generar más ítems que los que se van a utilizar en realidad en la versión final de dichos cuestionarios, para poder elegir una combinación óptima de ítems que pasan a constituir el contenido final del cuestionario. La selección de dichos ítems se realizó siguiendo criterios estadísticos y metodológicos principalmente, seleccionando únicamente aquellos ítems que cumplen unos determinados requisitos psicométricos (fiabilidad, validez y sensibilidad al cambio), a lo largo de un proceso largo y complejo. Esto fue precisamente lo que se llevó a cabo durante la segunda fase del proyecto: se redujo la cantidad inicial de ítems candidatos a formar parte del cuestionario, mediante un estudio de campo en el cual se administró el conjunto inicial de ítems a 250 pacientes oncológicos con astenia de diferente intensidad. Los sucesivos análisis realizados en el marco de esta recogida de datos permitieron seleccionar 12 ítems de entre los 75 enunciados iniciales. Estos 12 ítems se distribuyeron en 3 dimensiones: Actividades habituales, Limitaciones físicas, y Actitudes y creencias.

\section{Tercera fase. Validación (2005-2007)}

Una vez identificado el contenido del Cuestionario PERFORM, llegó el momento de comprobar que sus 12 ítems funcionaban realmente como una herramienta de medida. El proceso de validación es un requisito imprescindible antes de utilizar cualquier cuestionario o herramienta de medida centrada en el paciente, pues aporta evidencias de que el grupo de ítems que constituyen el cuestionario se comportan de forma coherente a como deberían comportarse si el cuestionario fuera efectivamente una herramienta de medida. La validación de un cuestionario suele centrarse en la evaluación de sus propiedades psicométricas: su fiabilidad, validez y sensibilidad al cambio.
La validación del Cuestionario PERFORM fue realizada con el objetivo de comprobar su correcto funcionamiento psicométrico. Para ello, el nuevo Cuestionario PERFORM fue administrado a una muestra de 437 pacientes oncológicos con astenia relacionada con el cáncer ${ }^{(46)}$. Durante esta fase, el cuestionario mostró propiedades psicométricas adecuadas y sólidas para ser utilizado en práctica clínica habitual(43).

Las principales características del cuestionario derivadas del estudio de validación pueden resumirse del siguiente modo:

a) El Cuestionario PERFORM mostró una buena viabilidad: el promedio de ítems perdidos por paciente fue inferior a 1 , y el $80 \%$ de pacientes completó la totalidad del cuestionario. El tiempo medio necesario para la administración del cuestionario fue inferior a 9 minutos. Más del $80 \%$ de pacientes consideraron el cuestionario fácil o muy fácil de completar. Las puntuaciones extremas fueron muy infrecuentes $(<5 \%)$, indicativo de que el cuestionario cubre bien el rango de intensidad del síntoma de la muestra estudiada.

b) La consistencia interna de la medida fue muy satisfactoria, tanto a nivel global como por dimensiones (valor alfa de Cronbach global: 0,935). Es decir, el comportamiento de los ítems del cuestionario es consistente entre sí.

c) La fiabilidad test-retest en el grupo de pacientes definidos como estables también fue muy satisfactoria, tanto a nivel global como por dimensiones (coeficiente de correlación intraclase global de 0,832). Es decir, las puntuaciones obtenidas con el cuestionario son similares cuando las circunstancias del paciente no han cambiado significativamente.

d) Además, el cuestionario presenta evidencias sólidas de validez de modo que se correlaciona de forma consistente 
con medidas de salud debidamente desarrolladas y validadas, como el cuestionario específico FACT-F (correlación 0,8) y el cuestionario genérico Perfil de Salud de Nottingham (correlación 0,69). Se correlaciona también de forma consistente con variables relacionadas con el estado de salud, como el índice Karnofsky, la intensidad de la astenia medida por escala analógica visual o el nivel de cuidados requeridos. Adicionalmente se asoció de forma consistente con los niveles de hemoglobina del paciente $(p=0,0163)$.

e) Finalmente, tanto la puntuación global como las puntuaciones de las dimensiones del Cuestionario PERFORM se mostraron sensibles a los cambios en el estado de salud autopercibido por el paciente, especialmente cuando este cambio suponía un empeoramiento.

\section{Aplicación del Cuestionario en la Práctica Clínica}

Dado que la publicación definitiva del cuestionario es muy reciente ${ }^{(43)}$, existen muy pocas experiencias de la aplicación del cuestionario en condiciones de práctica clínica habitual. En algunas pequeñas series aplicadas en mujeres con cáncer de mama sometidas a tratamiento sistémi$\mathrm{CO}^{(47,48)}$, presentaron mejores puntuaciones las pacientes con estadios tempranos frente a avanzados (45 vs 39), las sometidas a hormonoterapia o terapia biologica sola frente a quimioterapia (44 vs 47 vs 38), las menores de 60 años frente a las mayores de 60 (44 vs 34) y las pacientes con $\mathrm{Hb} \geq 12 \mathrm{~g} / \mathrm{dL}$ frente a $\mathrm{Hb}<12 \mathrm{~g} / \mathrm{dL}$ (44 vs 38), por lo que aunque el número de pacientes incluidas es pequeño, las puntuaciones obtenidas parecen relacionarse con el tipo de tratamiento, estadio, edad y niveles de $\mathrm{Hb}$. En otra serie de 50 pacientes tratados con radioterapia (RT) y diagnosticados de diversos tumores sólidos, globalmente no existieron diferencias sig- nificativas en las puntuaciones preRT vs postRT (48 vs 47; $\mathrm{p}=\mathrm{NS}$ ). Sin embargo, en el subgrupo de pacientes con cáncer de mama la puntuación media postRT fue significativamente inferior a la preRT $(p<0,05)$. Presentaron mejores puntuaciones postRT los pacientes con cáncer de próstata vs cáncer de mama (56 vs 39) y los pacientes con $\mathrm{Hb} \geq 12$ frente a $\mathrm{Hb}<12$ (51 vs 32). Por tanto, en pacientes con cáncer de mama sometidas a RT tras quimioterapia adyuvante, se observa un incremento significativo de la astenia inducida por RT. De igual modo los niveles de astenia se relacionaron nuevamente con los valores de Hemoglobina. No puede afirmarse que el tratamiento radioterápico impacte de forma negativa en la astenia en otros subgrupos de pacientes ${ }^{(49)}$.

En resumen, el cuestionario PERFORM ha sido desarrollado según los estándares internacionalmente aceptados para el desarrollo y la validación de herramientas de medida centradas en el paciente. Es una herramienta que ha probado ser viable, fiable, válida y sensible al cambio, y cuya extensión (sólo 12 ítems) la hace muy adecuada para su uso en la práctica clínica especializada. Los ítems del cuestionario se distribuyen en 3 dimensiones: actividades habituales (4 ítems), actitudes y creencias (4 ítems) y limitaciones físicas (4 ítems). Actualmente se dispone por tanto de un test ajustado a la realidad de los pacientes oncológicos con astenia en España.

\section{DESAFÍOS Y PERSPECTIVAS FUTURAS}

Tal y como se ha señalado, la astenia relacionada con el cáncer representa un problema frecuente en el paciente oncológico, con importante repercusión sobre su calidad de vida. A pesar de ello, son muchos los puntos que permanecen sin aclarar acerca de su fisiopatología y tratamiento. 
En el futuro, un conocimiento más amplio de los mecanismos que intervienen en el desarrollo de la astenia en el paciente con cáncer, permitirá diseñar terapias dirigidas o emplear otras intervenciones en la prevención de su aparición y manejo.

Por otra parte, y dada la relevancia del problema, es necesario implementar los instrumentos de evaluación y realizar ensayos bien diseñados que permitan conocer el verdadero valor de las medidas empleadas, dentro de las cuales, el cuestionario Perform constituye un test que por su brevedad y adecuado desarrollo psicométrico resulta en este momento la herramienta de elección.

Actualmente sólo las intervenciones no farmacológicas, especialmente el ejercicio físico, han demostrado un beneficio significativo en el manejo de la astenia relacionada con el cáncer en supervivientes. Sin embargo, los estudios actualmente en marcha ayudarán a diferenciar el tipo de ejercicio más eficaz, su intensidad, frecuencia y duración aconsejables, así como las posibles ventajas de la combinación con otras terapias.

En conclusión, el manejo de la astenia en supervivientes precisa una evaluación regular, con identificación de los posibles factores contribuyentes, potencialmente tratables, y el empleo de una serie de intervenciones, que incluyen la educación del paciente, la administración de fármacos y las terapias no farmacológicas.

\section{AGRADECIMIENTOS}

A los miembros del comité de expertos del Proyecto Perform (R. Colomer, J. Carulla, V. Valentín, J. Cassinello, J. GarcíaMata, P.Gascón y C.A.Rodríguez); de 3D Health Research (E.Baró y M.Herdman); y de Amgen (J. Sánchez y J.A. Gasquet), que trabajaron desde 2004 en el desarrollo y validación del cuestionario.

\section{REFERENCIAS BIBLIOGRÁFICAS}

1. National Comprehensive Cancer Network. Clinical Practice Guidelines in Oncology: Cancer Related Fatigue. Version 1.2011 [En línea] 2011 [Acceso 1 de marzo de 2012]. Disponible en: www.nccn.org/ professionals/physician_gls/f_guidelines. asp

2. Hofman M, Ryan JL, Figueroa-Moseley CD Morrow GR. Cancer-related fatigue: The scale of the problem. Oncologist 2007; 12 (Suppl 1):4-10. Doi:10.1634/ theoncologist.12-S1-4

3. Servaes P, Verghagen C, Bleijenberg G. Fatigue in cancer patients during and after treatment: prevalence, correlates and interventions. Eur J Cancer 2002; 38(1):2743. Doi:10.1016/S0959-8049(01)00332-X

4. Bower JE, Ganz PA, Desmond DA Bernaards $\mathrm{C}$, Rowland $\mathrm{JH}$, Meyerowitz BE, et al. Fatigue in long-term breast carcinoma survivors: A longitudinal investigation. Cancer 2006;106:751-8. Doi: 10.1002/ cncr.21671

5. Díaz N, Menjón S, Rolfo C, García-Alonso P, Carulla J, Magro A, et al. Patients' perception of cancer-related fatigue: results of a survey to assess the impact on their everyday life. Clin Transl Oncol 2008; 10:75357. Doi:10.1007/s12094-008-0282-x

6. Ryan JL, Carroll JK, Ryan EP. Karen M. Mustian, Kevin Fiscella, Gary R. Morrow. Mechanisms of cancer-related fatigue. Oncologist 2007; 12 (Suppl 1):2234. Doi:10.1634/theoncologist.12-S1-22

7. Barsevick A, Frost M, Zwinderman A, Hall P, Halyard M, GENEQOL Consortium. I'm so tired: Biological and genetic mechanisms of cancer-related fatigue. Qual Life Res 2010; 19 (10):1419-27. Doi:10.1007/ s11136-010-9757-7

8. Sephton S, Spiegel D. Circadian disruption in cancer: a neuroendocrine-immune pathway from stress to disease? Brain Behav Immun 2003;17:321-8. Doi: 10.1016/ S0889-1591(03)00078-3, 
9. Bower JE, Ganz PA, Desmond KA, Fahey JL, Cole SW. T-cell homeostastis in breast cancer survivors with persistent fatigue. J Natl Cancer Inst 2003; 95:1165-8. Doi:10.1093/jnci/djg0019

10. Glaspy J, Degos L, Dicato M George D. Demetri GD. Comparable efficacy of epoetin alfa for anemic cancer patients receiving platinum- and nonplatinum-based chemotherapy: A retrospective subanalysis of two large, community-based trials. Oncologist 2002; 7:126-35. Doi:10.1634/ theoncologist.7-2-126

11. Littlewood TJ, Bajetta E, Nortier JW Vercammen E, Rapoport B, Epoetin Alfa Study Group. Effects of epoetin alfa on hematologic parameters and quality of life in cancer patients receiving nonplatinum chemotherapy: Results of a randomized, double-blind, placebo-controlled trial. J Clin Oncol 2001; 19:2865-74.

12. Visser MR, Smets EM. Fatigue, depression and quality of life in cancer patients: How are the related? Support Care Cancer 1998; 6:101-8. Doi:10.1007/s005200050142

13. Barsevick AM, Dudley W, Beck S Sweeney C, Whitmer K, Nail L.A randomized clinical trial of energy conservation for patients with cancer-related fatigue. Cancer 2004;100:1302-1310. Doi: 10.1002/ cncr.20111

14. Graydon JE, Bubela N, Irvine D Vincent L. Fatigue-reducing strategies used by patients receiving treatment for cancer. Cancer Nurs 1995; 18:23-8. Doi:10.1097/00002820199502000-00004

15. Chang J, Couture F, Young S McWatters KL, Lau CY. Weekly epoetin alfa maintains hemoglobin, improves quality of life, and reduces transfusion in breast cancer patients receiving chemotherapy. J Clin Oncol 2005; 23(12):2597-605.

16. Vansteenkiste J, Pirker R, Massuti B Barata F, Font A, Fiegl $M$ et al. Double-blind, placebo-controlled, randomized phase III trial of darbepoetin alfa in lung cancer patients receiving chemotherapy. J Natl Cancer
Inst 2002; 94(16):1211-20. Doi:10.1093/ jnci/94.16.1211

17. Butler JM, Jr., Case LD, Atkins J Frizzell B, Sanders G, Griffin P, et al. A phase III, double-blind, placebo-controlled prospective randomized clinical trial of d-threomethylphenidate $\mathrm{HCl}$ in brain tumor patients receiving radiation therapy. Int J Radiat Oncol Biol Phys 2007;69:1496-1501.

18. Mar Fan HG, Clemons M, Xu W Chemerynsky I, Breunis H, Braganza S, et al. A randomised, placebo-controlled, double-blind trial of the effects of d-methylphenidate on fatigue and cognitive dysfunction in women undergoing adjuvant chemotherapy for breast cancer. Support Care Cancer 2008;16:577-83. Doi: 10.1007/s00520-007-0341-9

19. Moraska AR, Sood A, Dakhil SR Sloan JA, Barton D, Atherton PJ, et al. Phase III, randomized, double-blind, placebo controlled study of long-acting methylphenidate for cancer-related fatigue: North Central Cancer Treatment Group NCCTG-N05C7 trial. J Clin Oncol 2010; 28:3673-9.

20. Lower EE, Fleishman S, Cooper A Zeldis J, Faleck H, Yu Z, et al. Efficacy of dexmethylphenidate for the treatment of fatigue after cancer chemotherapy: A randomized clinical trial. J Pain Symptom Manage 2009;38:650-62. Doi: 10.1016/j. jpainsymman.2009.03.011,

21. Hanna A, Sledge G, Mayer ML Hanna N, Einhorn L, Monahan P, et al. A phase II study of methylphenidate for the treatment of fatigue. Support Care Cancer 2006;14:210-215. Doi: 10.1007/s00520005-0857-9

22. Lower EE, Fleishman S, Cooper A, Jerome Zeldis, Herbert Faleck, Zhinuan Yu, et al. Efficacy of dexmethylphenidate for the Treatment of fatigue after cancer chemotherapy: A randomized trial. J Pain Symptom Manage 2009; 38:650-62. Doi:10.1016/j.jpainsymman.2009.03.011

23. Jean-Pierre $P$, Morrow GR, Roscoe JA Heckler C, Mohile S, Janelsins M, et al. 
Phase 3 randomized, placebo-controlled, double-blind, clinical trial of the effect of modafinil on cancer-related fatigue among 631 patients receiving chemotherapy: A University of Rochester Cancer Center Communnity Clinical Oncology Program Research base study. Cancer 2010; 116:3513-20. Doi:10.1002/cncr.25083

24. Morrow GR, Hickok JT, Roscoe JA Raubertas RF, Andrews PL, Flynn PJ, et al. Differential effects of paroxetine on fatigue and depression: a randomized, double-blind trial from the University of Rochester Cancer Center community Clinical Oncology Program. J Clin Oncol 2003; 21:4365-41.

25. Roscoe JA, Morrow GR, Hickok JT Mustian KM, Griggs JJ, Matteson SE, et al. Effect of paroxetine hydrochloride (Paxil) on fatigue and depression in breast cancer patients receiving chemotherapy. Breast Cancer Res Treat 2005; 89:243-9. Doi:10.1007/ s10549-004-2175-1

26. Cullum JL, Wojciechowski AE, Pelletier G Simpson JS. Bupropion sustained release treatment reduces fatigue in cancer patients. Can J Psychiatry 2004;49:139-44.

27. Moss EL, Simpson JS, Pelletier G, Forsyth P. An open-label study of the efects of bupropion SR on fatigue, depression and quality of life of mixed-site cancer patients and their partners. Psychooncology 2006; 15:259-67. Doi: 10.1002/pon.952

28. Bruera E, Roca E, Cedaro L Carraro S, Chacon R. Action of oral methylprednisolone in terminal cancer patients: A prospective randomized double-blind study. Cancer Treat Rep 1985; 69:751-4.

29. Bruera E, Ernst S, Hagen N Spachynski K, Belzile M, Hanson J et al. Effectiveness of megestrol acetate in patients with advanced cancer: A randomized, doubleblind, crossover study. Cancer Prev Control 1998; 2:74-8.

30. De Oliveira Campos MP, Riechelmann R, Martins LC Hassan BJ, Casa FB, Del Giglio A. Guarana (Paullina cupana) improves fatigue in breast cancer patients un- dergoing systemic chemotherapy. J Altern Complement Med 2011;17(6):505-12. doi:10.1089/acm.2010.0571.

31. Brown JC, Huedo-Medina TB, Pescatello LS Pescatello SM, Ferrer RA, Johnson BT, et al. Efficacy of exercise interventions in modulating cancer-related fatigue among adult cancer survivors: a meta-analysis. Cancer Epidemiol Biomarkers Prev 2011; 20(1):123-33. DOI:10.1158/1055-9965. EPI-10-0988

32. McNeely $\mathrm{ML}$, Campbell $\mathrm{KL}$, Rowe $\mathrm{BH}$ Klassen TP, Mackey JR, Courneya KS. Effects of exercise on breast cancer patients and survivors: A systematic review and meta-analysis. CMAJ 2006;175:34-41. Doi:10.1503/cmaj.051073

33. Dimeo FC, Tiolmann MH, Bertz H Kanz L, Mertelsmann R, Keul J. Aerobic exercise in the rehabilitation of cancer patients after high dose chemotherapy and autologous peripheral stem cell transplantation. Cancer 1997;79:171722. Doi: $10.1002 /(\mathrm{SICl}) 1097-$ $0142(19970501) 79: 9<1717:$ :AID CNCR12>3.0.CO;2-0

34. Schmitz KH, Courneya KS, Matthews C, Demark-Wahnefried W, Galvão DA, Pinto BM, et al. American College of Sports Medicine roundtable on exercise guidelines for cancer survivors. Med Sci Sports Exerc 2010;42:1409-26. doi: 10.1249/ MSS.0b013e3181e0c112

35. Lindemalm C, Strang P, Lekander M. Support group for cancer patients. Does it improve their physical and psychological wellbeing? A pilot study. Support Care Cancer 2005;13:652-57. Doi: 10.1007/ s00520-005-0785-8

36. Dolbeault $S$, Cayrou $S$, Bredart A Viala AL, Desclaux B, Saltel P, et al. The effectiveness of a psycho-educational group after earlystage breast cancer treatment: Results of a randomized French study. Psychooncology 2009;18:647-56. Doi: 10.1002/pon.1440

37. Van Weert E, May AM, Korstjens I Post WJ, van der Schans CP, van den Borne B, 
Mesters I, Ros WJ, Hoekstra-Weebers JE. Cancer-related fatigue and rehabilitation: A randomized controlled multicenter trial comparing physical training combined with cognitive-behavioral therapy with physical training only and with no intervention. Phys Ther 2010,90(10):1413-25. Doi: 10.2522/ptj.20090212

38. Mustian KM, Morrow GR, Carroll JK Mustian KM, Morrow GR, Carroll JK. Integrative nonpharmacologic behavioral interventions for the management of cancer-related fatigue. Oncologist 2007; 12 (suppl 1): 5267. Doi: 10.1634/theoncologist.12-S1-52.

39. Jean-Pierre P, Figueroa-Moseley CD, Kohli S Fiscella K, Palesh OG, Morrow GR. Assessment of cancer-related fatigue: implications for clinical diagnosis and treatment. Oncologist 2007;12 (suppl 1): 1121. Doi: 10.1634/theoncologist.12-S1-11

40. Minton O, Stone P. A systematic review of the scales used for the measurement of cancer-related fatigue (CRF). Ann Oncol 2009; 20:17-25. Doi: 10.1093/annonc/ mdn537

41. Glauss A. Assessment of fatigue in cancer and non-cancer patients and in healthy individuals. Support Car Cancer 1993; 1:305-15.

42. Baró E, Carulla J, Cassinello J Colomer R, Mata JG, Gascón P, et al. Psychometric properties of the Perform Questionnaire: A brief scale for assessing patient perceptions of fatigue in cancer. Support Care Cancer 2011; 19(5):657-66. Doi: 10.1007/ s00520-010-0878-x

43. Herdman M, Fox-Rushby J, Badia X. A model of equivalence in the cultural adaptation of HRQoL instruments: The universalist approach. Qual Life Res. 1998;7(4):32335. Doi: 10.1023/A:1024985930536

44. Scientific Advisory Committee of the Medical Outcomes Trust. Assessing health status and quality-of-life instruments: Attributes and review criteria. Quality of Life Research. 2002;11:193-205. Doi: 10.1023/A:1015291021312

45. Baró E, Carulla J, Cassinello J, Colomer R, Mata JG, Gascón P, et al. Development of a new questionnaire to assess patient perceptions of_cancer-related_fatigue: item generation and item reduction. Value Health. 2009; 12(1):130-8. Doi:10.1111/ j.1524-4733.2008.00426.x

46. Rodríguez C, Gascón P, García-Mata J, Colomer R, Cassinello J, Carulla J, et al. Validation of a new questionnaire to assess the patient perception of cancer-related fatigue (CRF): the Perform Questionnaire (PQ). Journal of Clinical Oncology, 2007 ASCO Annual Meeting Proceedings Part I. Vol 25, No. 18 S (June 20 Supplement), 2007: 19533.

47. Rodríguez CA, Viotti F, Gómez A, Seijas A, López R, Del Barco E, et al. Evaluación de la astenia en pacientes con cáncer de mama y tratamiento sistémico. Resultados preliminares. III Congreso SEOM de Cuidados Continuos en Oncología Médica 2008; Abs P-28.

48. Rodríguez CA, Viotti F, El-Hallaj M, Reguero $\mathrm{V}$, Leno R, Bratos R, et al. Aplicación del cuestionario perform en la evaluación de la astenia en pacientes con cáncer de mama y tratamiento sistémico en condiciones de práctica clínica asistencial. XII Congreso de la Sociedad Española de Oncología Médica 2009; Abs P-126.

49. Alonso R, Rodríguez CA, Soria P, Vidal $R$, Galeazzi V, Rivas B, et al. Evaluación de la astenia durante el tratamiento con radioterapia (RT). Aplicación del cuestionario Perform. Resultados preliminares. XIII Congreso de la Sociedad Española de Oncología Médica 2011; Abs P-97. 
